\title{
"Pornographic binges" as a key characteristic of males seeking treatment for compulsive sexual behaviors: Qualitative and quantitative 10-week-long diary assessment
}

\author{
MAŁGORZATA WORDECHA ${ }^{1}$, MATEUSZ WILK $^{1,2}$, EWELINA KOWALEWSKA ${ }^{1,3}$, MACIEJ SKORKO $^{1}$, ADAM ŁAPIŃSKI ${ }^{4}$ \\ and MATEUSZ GOLA ${ }^{1,5 *}$ \\ ${ }^{1}$ Clinical Neuroscience Laboratory, Institute of Psychology, Polish Academy of Sciences, Warsaw, Poland \\ ${ }^{2}$ Faculty of Mathematics and Computer Science, Nicolaus Copernicus University, Torun, Poland \\ ${ }^{3}$ Department of Psychology, University of Social Sciences and Humanities, Warsaw, Poland \\ ${ }^{4}$ Predict Watch, Bialystok, Poland \\ ${ }^{5}$ Swartz Center for Computational Neuroscience, Institute for Neural Computation, University of California San Diego, \\ San Diego, CA, USA
}

(Received: September 6, 2017; revised manuscript received: March 23, 2018; second revised manuscript received: March 24, 2018; accepted: March 25, 2018)

\begin{abstract}
Background and aims: Compulsive sexual behaviors (CSBs) are an important clinical and social issue. Despite the increasing number of studies, some of CSB's aspects remain under-investigated. Here, we explore the nature of CSB, such as binge pornography use and masturbation (PuM), and verify the correspondence between self-perceived factors leading to such behavior with its measures obtained in a diary assessment. Methods: Semi-structuralized interviews with nine treatment-seeking males aged 22-37 years $(M=31.7, S D=4.85)$ were followed by a questionnaire and a 10-week-long diary assessment, allowing us to acquire real-life daily patterns of CSB. Results: Six out of nine subjects experienced binge (multiple hours or times a day) PuM. All subjects presented a high level of anxiety and perceived PuM as a way to regulate mood and stress. Data collected in the diary assessment uncovered a high diversity in the patterns of sexual behaviors (such as frequency of regular and binge PuM) and its correlates. Binge PuM was related to decreased mood and/or increased stress or anxiety. The causal relation between these correlates remains undetermined. Discussion and conclusions: Binge PuM seems to be one of the most characteristic behavior among males who are seeking treatment for CSB and is related to the feeling of losing control over one's sexual activity. CSB individuals indicate a variety of binge triggers. Also, diary assessment data indicate that specific correlates of binge PuM (decreased mood, increased stress, and anxiety) differ between subjects. It suggests the existence of significant individual differences in binge PuM behaviors, and a need to study these differences, as it may help guide personalized treatment.
\end{abstract}

Keywords: compulsive sexual behaviors, hypersexuality, pornography, masturbation, diary assessment

\section{INTRODUCTION}

For some people, compulsive sexual behaviors (CSBs) are a reason to seek treatment (Gola, Lewczuk, \& Skorko, 2016; Lewczuk, Szmyd, Skorko, \& Gola, 2017). Given this reality, the number of studies on this topic has increased substantially (Gola, Wordecha, Marchewka, \& Sescousse, 2016; Kraus, Voon, \& Potenza, 2016a), and there is an ongoing discussion about including CSB in the next edition of the International Classification of Diseases (ICD; Gola \& Potenza, 2018; Kraus et al., 2018; Potenza, Gola, Voon, Kor, \& Kraus, 2017; Prause, Janssen, Georgiadis, Finn, \& Pfaus, 2017; World Health Organization [WHO], 2018). The most commonly reported symptoms concern time spent on pornography viewing (mainly on the Internet) and excessive masturbation (Gola, Lewczuk, et al., 2016; Kafka, 2010; Reid, Garos, \& Carpenter, 2011; Stein, Black, Shapira, \& Spitzer, 2001).
Other reported types of behaviors include risky casual sexual relations, anonymous sex, and use of paid sexual services (Kraus, Voon, \& Potenza, 2016a).

Despite the ongoing debate on how to conceptualise CSB (Kor, Fogel, Reid, \& Potenza, 2013; Kraus, Voon, \& Potenza, 2016b; Ley, Prause, \& Finn, 2014; Potenza et al., 2017), World Health Organization included CSB in the proposal for the upcoming ICD-11 (WHO, 2018) as an impulse control disorder (Kraus et al., 2018) with symptomatology very similar to that previously proposed by Kafka (2010). According to these criteria, we may recognize

\footnotetext{
* Corresponding author: Mateusz Gola; Clinical Neuroscience Laboratory, Institute of Psychology, Polish Academy of Sciences, 1 Jaracza St. 00-378 Warsaw, Poland; Phone: +48 2258313 80; Fax: +48 2258313 81; E-mail: mgola@psych.pan.pl
}

This is an open-access article distributed under the terms of the Creative Commons Attribution-NonCommercial 4.0 International License, which permits unrestricted use, distribution, and reproduction in any medium for non-commercial purposes, provided the original author and source are credited, a link to the CC License is provided, and changes - if any - are indicated. 
CSB if (a) over a period of at least 6 months, at least four out of five of the following symptoms are observed:

1. excessive time spent on sexual fantasies, urges, or behaviors repeatedly interferes with other important (non-sexual) goals, activities, and obligations, i.e., pornography viewing has become a central interest in one's life, so that family duties or work obligations are neglected;

2. the subject repetitively engages in these sexual activities in response to dysphoric emotional states, i.e., sexual activity has become a rigid strategy of mood regulation;

3. and/or a response to stressful situations, e.g., during stressful events at work;

4. despite repeated attempts, the subject fails to control or significantly reduce these sexual activities, i.e., the subject makes numerous unsuccessful attempts to limit problematic activities, but invariably loses control over them after a couple of days;

5. the subject continues these sexual activities despite the risk of physical or emotional harm to self or to others, i.e., engaging in frequent sexual behavior despite serious consequences for relationships (e.g., break-up) or threat of job loss.

(b) The frequency and intensity of these sexual activities lead to clinically significant personal distress or dysfunction in important aspects of life. (c) These sexual activities were not the result of exogenous substance use (e.g., drug abuse or medication).

However, while Kafka's (2010) definition of CSB is commonly accepted, it does not propose any mechanism underlying CSB. Recent studies suggest that CSBs are related to increased sensitivity for erotic rewards (Brand, Snagowski, Laier, \& Maderwald, 2016; Kraus et al., 2016b; Voon et al., 2014) or cues predicting such rewards (Gola, Wordecha, et al., 2017). Others indicate increased cue-conditioning for erotic stimuli (Klucken, Wehrum-Osinsky, Schweckendiek, Kruse, \& Stark, 2016) or increased anxiety (Gola, Miyakoshi, \& Sescousse, 2015; Gola \& Potenza, 2016) among individuals with CSB. Reid also noted that hypersexual patients often experience negative emotions and stress, more intense shame, and have a lower level of self-compassion (Reid, Stein, \& Carpenter, 2011; Reid, Temko, Moghaddam, \& Fong, 2014).

The multiplicity and diversity of the factors described above give rise to at least three important questions: (a) How do individuals seeking treatment perceive key factors leading to CSB?, (b) Which of those self-perceived factors indeed correlate with data assessed in everyday life situations?, and (c) How homogeneous are these factors in CSB?

Such questions can be answered with qualitative data (i.e., collected during structuralized clinical interviews, as in Carpenter, Reid, Garos, \& Najavits, 2013) and with a quantitative approach, using the diary assessment method (Kashdan et al., 2013). Diary assessment is considered to be highly ecologically valid for measuring individual daily states (e.g., anxiety level, mood, and sexual arousal) and activities (e.g., sexual behaviors). In this study, we decided to combine both qualitative and diary assessment approaches to examine the factors related to CSB in subjects voluntarily seeking treatment for CSB.
As there are no quantitative norms for sexual behaviors (Gola, Lewczuk, et al., 2016), CSBs are usually defined by descriptive symptoms, reflecting the subjective loss of control over sexual activity (Gola \& Potenza, in press; Kafka, 2010; Kraus et al., 2018). We may try to find some quantitative factors underlying this subjective phenomenon, such as excessive amount of time spent on sexual activity (i.e., masturbation and pornography use interfering with one's job) or the wrong places where one engages in sexual activity (i.e., in public places or restrooms). One such measurable pattern of addictive behavior is binging - a repetitive, continuous, and massive behavior - often leading to a subjective feeling of the loss of control. Binges have been extensively described in substance-use disorders, such as alcohol-use disorder (Rolland \& Naassila, 2017).

Patients seeking treatment for CSB also report binge sexual activity (Gola, Wordecha, et al., 2017), and often mention that this is the most extreme form of the loss of control over one's behavior (Lewczuk et al., 2017). Usually, such binges involve many hours of pornography viewing (continuously or multiple times a day), accompanied by multiple masturbations. Binge pornography use has not been described in scientific literature in sufficient detail. Therefore, we propose to take a closer look at this aspect of CSB and to find out how common a symptom it is among individuals seeking treatment for CSB. Thus we aim to (a) examine how subjects seeking treatment for CSB describe factors related to their CSBs, (b) determine how it corresponds to data collected in the diary assessment, and (c) investigate whether those factors are homogeneous across all individuals with CSB and which of them are related to binge and non-binge sexual activities.

\section{METHODS}

\section{Participants}

Our group consisted of nine CSB males aged 22-37 years $(M=31.7, S D=4.85$; Table 1$)$. All patients suffered from recurrent sexual fantasies/behaviors and admitted that their sexual behavior resulted in the mishandling of important life duties. All patients noticed a gradual progression of the problem and admitted using sexual behaviors (mostly pornography viewing accompanied by masturbation) to cope with stressful life events. Each of the patients reported multiple attempts to limit or terminate CSB. Usually, effects were poor and temporary, but some reported longer periods of sexual abstinence (several months up to 1 year) followed by relapses. Almost all subjects had a history of previous CSB treatment. During the study, one subject (Subject B) was maintaining abstinence from PuM (he had almost daily sexual intercourse with spouse).

\section{Recruitment procedure}

All subjects were recruited among patients seeking treatment for CSB in sexual health treatment centers in Warsaw (Poland). All subjects met at least four out of five CSB criteria according to Kafka (described in the 


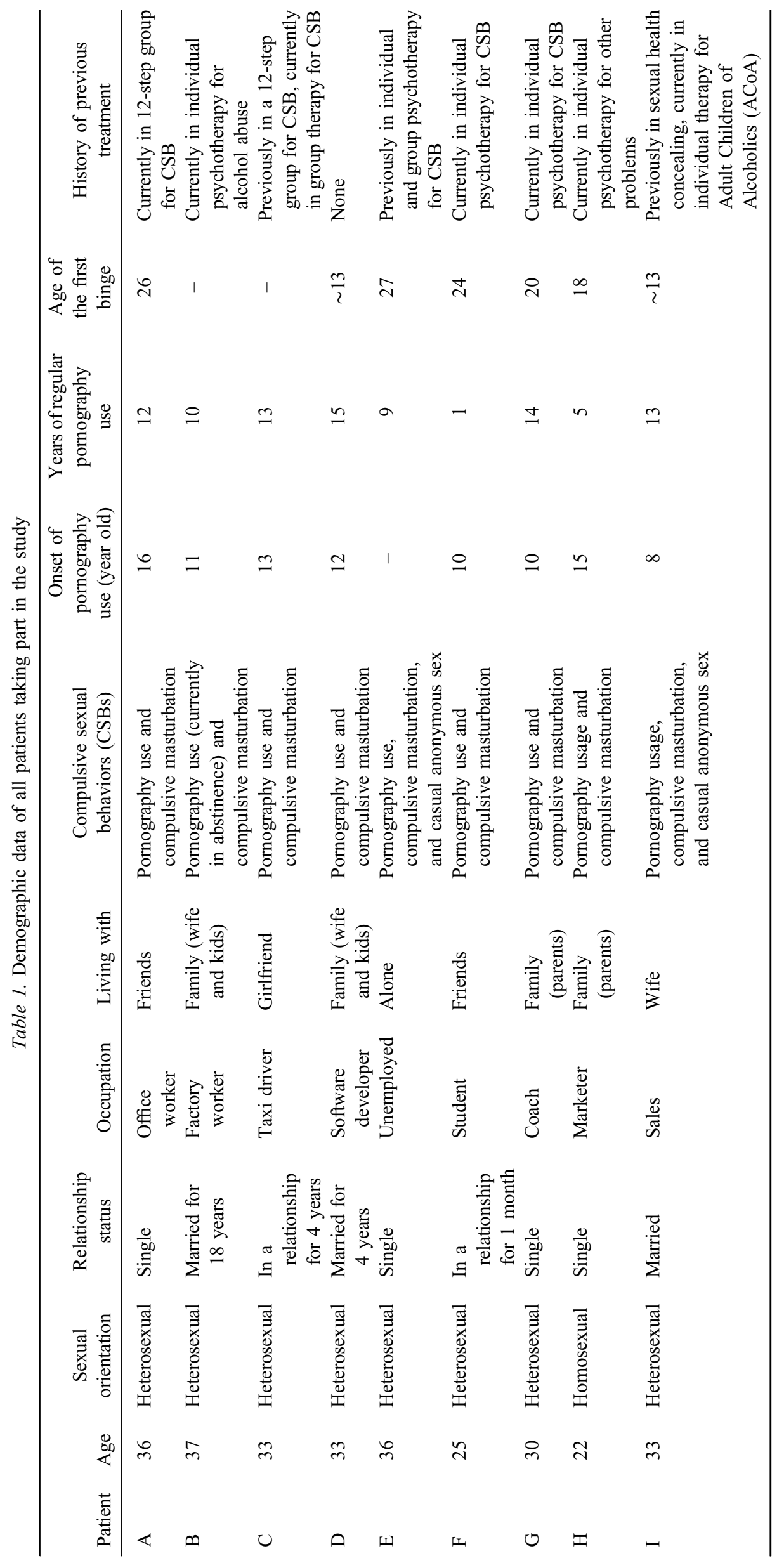


"Introduction" section). Also, all of them took part in at least six sessions of treatment for CSB after being enrolled to this study, which demonstrates their real intent of limiting problematic pornography use.

\section{Measures}

We conducted an hour-long semi-structured interview (Supplementary Table S1) to assess the most common symptoms of CSB (including binge PuM), self-perceived underlying psychological mechanisms, and factors related to CSB. After this interview, subjects participated in a diary study lasting 10 weeks (70 days), using a web-based application accessible through smartphones or personal computers (Figure 1). Diary assessment partially overlapped with onset of treatment, thus data reported in diaries may have been influenced by the treatment. Using 10-point scales, we assessed daily measures of sexual arousal, anxiety, stress, and mood. We also assessed sexual behaviors, such as daily time spent watching pornography, the number of masturbation sessions, or the number of sexual intercourses. Subjects were requested to fill the diary once a day, which usually took 3-5 min. However, only seven out of nine participants provided requested information, and the average duration of episodes when diary entries were not made was 2.75 days with $\min =1$ day and $\max =32$ days. Detailed information is provided in the Supplementary Table S2. Records with missing data were excluded from the calculations of average values and standard deviations of the factors under study. Reported confidence intervals were estimated using the moving block bootstrap method with block size $=3$, applied to full data sets (including missing data).

We also collected questionnaire measurements. Severity of CSBs was assessed with the Sexual Addiction Screening Test - Revised (SAST-R; Carnes, Green, \& Carnes, 2010; Gola, Skorko, et al., 2017) and Brief Pornography Screener (BPS; Kraus et al., 2017). The BPS Questionnaire is a five-item scale, measuring the severity of problematic pornography use. Severity of obsessive-compulsive disorder (OCD) symptoms was assessed with the ObsessiveCompulsive Inventory - Revised (OCI-R; Foa et al., 2002). Anxiety level was measured with the State-Trait Anxiety Inventory - State (STAI-S; Sosnowski \& Wrześniewski, 1983), which allowed us to measure anxiety as a state (STAI-S) and trait (STAI-T). We also used Hospital Anxiety and Depression Scale (Zigmond \& Snaith, 1983) for assessing anxiety and depression symptoms. Impulsivity was

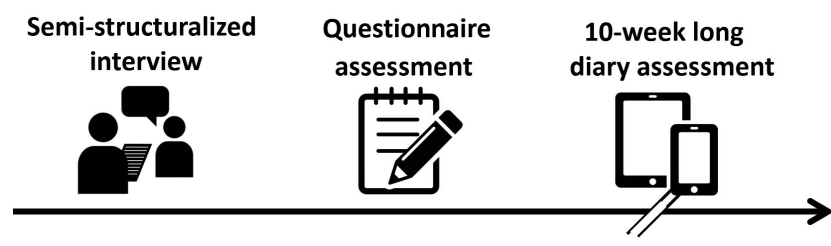

Figure 1. Schematic presentation of the research methods. All subjects were first interviewed with a semi-structuralized interview (Supplementary Table S1), and then took part in a questionnaire assessment (Supplementary Table S3) and 10-week-long web-based diary assessment assessed with Monetary Choice Questionnaire (Kirby \& Maraković, 1996), a set of 27 choices, in which participants should indicate if they would prefer a smaller monetary reward today or a larger one in the future (after a specific number of days).

\section{Ethics}

The study was approved by the Ethics Committee of the Institute of Psychology, Polish Academy of Sciences (in accordance with the Declaration of Helsinki) and all participants gave their written consent.

\section{RESULTS}

\section{Questionnaire measurements}

All patients achieved high scores in SAST-R and BPS. The majority of patients also obtained high scores on the subscales of depression and anxiety of the Hospital Anxiety and Depression Scale (Zigmond \& Snaith, 1983) and the STAI (Sosnowski \& Wrześniewski, 1983), as presented in Supplementary Table S3. Only two subjects exceed the threshold for the compulsivity dimension, measured with the OCI-R (Foa et al., 2002). Detailed results are presented in Supplementary Table S3.

\section{Self-declared and diary-assessed characteristics of CSB}

All subjects declared compulsive PuM as the most significant reason for seeking treatment. Only two individuals reported casual sexual relations as an additional problematic behavior. One patient sought treatment, despite 6.5 months of sexual abstinence prior to the study. For eight out of nine patients, this was not the first attempt to treat CSB (Table 1).

Despite multiple attempts to limit pornography use, the average time devoted to pornography viewing per week was $2.96 \mathrm{hr}$, as declared by the subjects in the questionnaires administered after the interviews. According to data collected over 10 weeks of the diary assessment, however, it was $1.57 \mathrm{hr}(S D=2.05 \mathrm{hr})$. We observed large interindividual variability of pornography use (from 0.5 to $8 \mathrm{hr}$ per week, as declared in interviews and from 0 to $6.01 \mathrm{hr}$ per week, as declared in the diary assessment; Table 2).

Data collected in the diary assessment revealed that pornography viewing was mostly accompanied by masturbation (Figure 2), which was in line with declarative data. During the interviews, six subjects reported that pornography viewing is always accompanied by masturbation, and three subjects reported that masturbation usually (but not always) is accompanied by pornography watching. However, masturbation without pornography viewing is usually accompanied by sexual memories of previously watched pornographic materials or fantasies about real people. One patient claimed that masturbation without pornography does not lead to a climax in his case.

During the interviews, seven out of nine patients reported at least one experience of binge pornography watching. Binges had the form of either continuous pornography watching accompanied by multiple masturbations for a few 


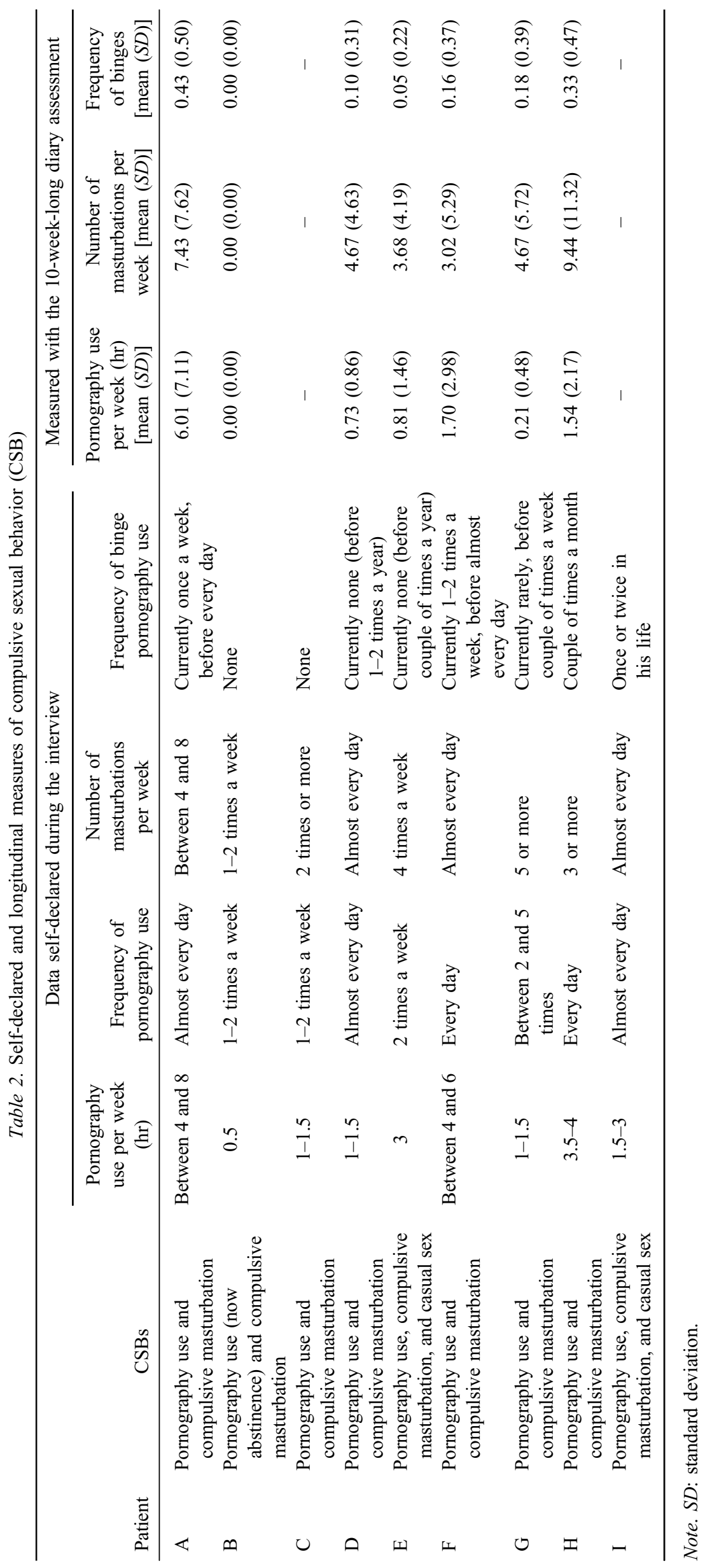




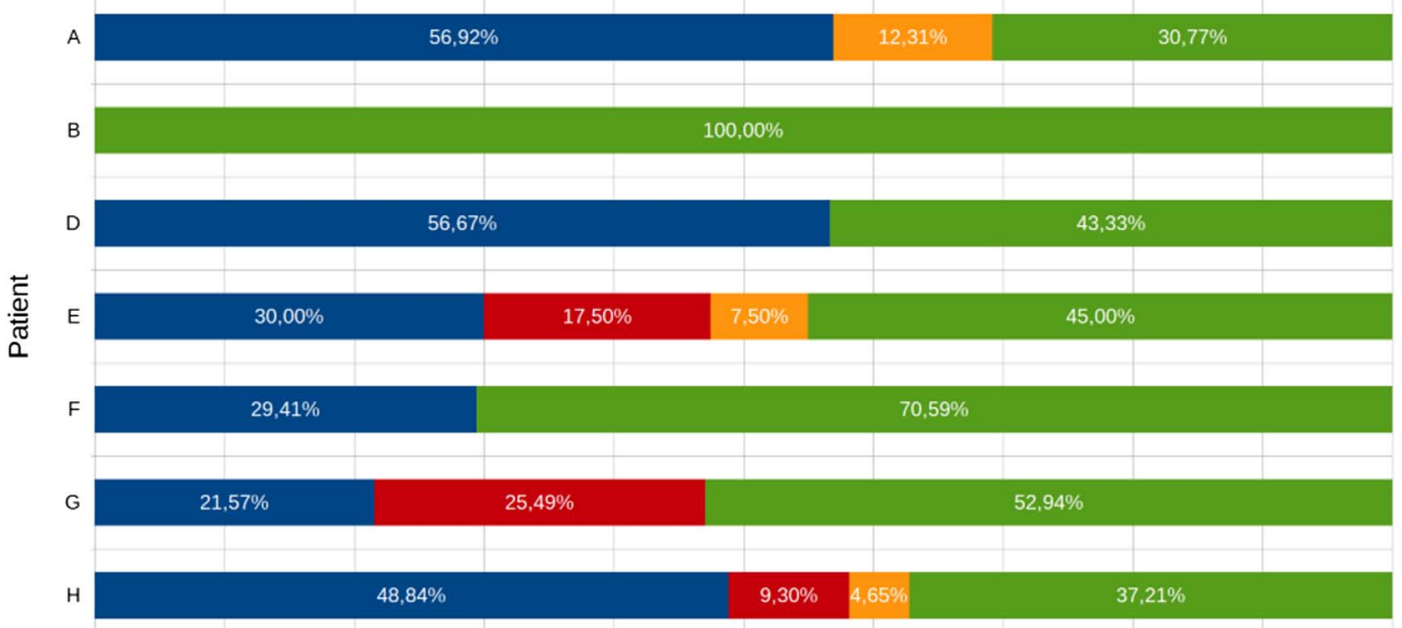

Neither masturbation nor pornography

- Masturbation and no pornography m Pornography and no masturbation

Masturbation and pornography

Figure 2. Distribution of daily combinations of pornography viewing and masturbation in data collected in the diary assessment - data from the diary measurement (100\% is equivalent to all days of diary assessments after exclusion of missing data)

hours in a row (usually $>6 \mathrm{hr}$ with less than 30 min breaks) or multiple episodes ( $>4$ per day, lasting for $0.5-1 \mathrm{hr}$ ) of pornography viewing a day accompanied by masturbation. One subject (Subject B), who reported 6.5 months of sexual abstinence, did not report any experience of binge pornography viewing, whereas Subject $\mathrm{C}$ reported a maximum of two episodes of pornography viewing and masturbation per day, which he did not consider a binge.

For the purpose of data analysis, we adopted an a priori definition of "binge," based on data from our previous studies (Gola, Kowalewska, Wierzba, Wordecha, \& Marchewka, 2015; Gola, Lewczuk, et al., 2016; Gola, Skorko, et al., 2017; Gola, Wordecha, et al., 2017; Lewczuk et al., 2017) indicating that in the control group (nontreatment seeking Polish males), the average number of masturbations per week is $2.3-2.5$ and the average time spent on pornography viewing is $50 \mathrm{~min} /$ week. Control subjects in our previous studies as a lifetime maximum number of masturbations and pornography watching per day reported an average of 3.1 and $70 \mathrm{~min}$. Both (maximal episodes of masturbation and pornography viewing) were considered by control individuals as binge sexual activities. For the purpose of this study based on our previous observations, we arbitrarily set a threshold, assuming that more than two masturbations per day and a single pornography session lasting longer than $1 \mathrm{hr}$ qualify as binge activity. Although these thresholds appeared to match self-declared data during the interview and data assessed with diary methods (Table 2), they should be carefully verified among different populations. Here, we study individuals who are already willing to terminate pornography viewing and expand considerable effort on this goal.

\section{Factors related to PuM}

Each of the patients reported multiple attempts to limit or terminate pornography use. In most cases, the outcomes were poor and temporary, but some reported periods of abstention from pornography lasting from several weeks up to 1 year, always followed by relapses. For one patient, a period without pornography lasting a few weeks was related to high work load; and to another, it increased social activities. One of the patients reported meditation to be temporarily helpful in limiting pornography use.

During the interviews, eight of nine patients were able to identify their patterns of PuM, indicating certain places, situations, emotions, and/or thoughts. The most common place of pornography use was the patient's home. And the most common situation was being alone. Four subjects also reported frequent pornography watching in public places, mostly at work. Four other patients said that they usually used pornography before or after working hours.

The majority of the patients reported negative emotions after pornography watching: stress (five subjects), anger (three), anxiety and tension (three), loneliness (two), low self-esteem (one), a sense of failure (three), and tiredness (two).

Most of the patients had difficulty in identifying the exact triggers of pornography watching. One patient identified increased stress and imagined risk of failure as the most common self-perceived factor leading to sexual activity. Another patient noted strong anger as a factor triggering PuM. One subject distinguished two types of masturbation he had engaged in: (a) related to sexual desire and (b) for anxiety reduction. He also noticed that the latter was more common in his case. Only one patient described pornography watching as a pleasurable "prize" he gave himself for personal achievements.

To investigate which factors are related to $\mathrm{PuM}$, we analysed the data obtained in diary assessments, comparing reports from the days with masturbation and pornography use with reports from the days without such activities. We examined differences between average levels of several factors, assessed with diaries, namely mood, tiredness, 
Table 3. Average data from the 10-week-long diary assessment (scale: 1-10)

\begin{tabular}{llcccc}
\hline Patient & $\begin{array}{c}\text { Mood } \\
{[\text { mean }(S D)]}\end{array}$ & $\begin{array}{c}\text { Tiredness } \\
{[\text { mean }(S D)]}\end{array}$ & $\begin{array}{c}\text { Stress level } \\
{[\text { mean }(S D)]}\end{array}$ & $\begin{array}{c}\text { Anxiety level } \\
{[\text { mean }(S D)]}\end{array}$ & $\begin{array}{c}\text { Sexual arousal } \\
{[\text { mean }(S D)]}\end{array}$ \\
\hline A & $4.92(1.56)$ & $6.23(1.63)$ & $5.86(1.63)$ & $5.54(1.91)$ & $2.42(1.43)$ \\
B & $5.52(1.99)$ & $6.43(1.57)$ & $4.43(2.06)$ & $4.14(2.08)$ & $4.71(1.82)$ \\
D & $5.3(1.58)$ & $5.23(1.74)$ & $4.5(2.01)$ & $3.07(2.26)$ & $3.7(1.21)$ \\
E & $7.2(0.69)$ & $4.9(1.55)$ & $4.45(1.08)$ & $3.35(1.23)$ & $4.0(0.88)$ \\
F & $6.35(1.43)$ & $4.8(1.81)$ & $3.1(1.5)$ & $2.2(1.04)$ & $5.1(1.79)$ \\
G & $6.0(1.6)$ & $6.47(1.77)$ & $5.51(1.87)$ & $4.76(2.17)$ & $4.9(2.04)$ \\
H & $4.3(2.18)$ & $6.23(1.76)$ & $4.74(1.98)$ & $4.88(2.2)$ & $3.88(1.99)$ \\
Group & $5.66(0.96)$ & $5.76(0.75)$ & $4.66(0.89)$ & $3.99(1.17)$ & $4.10(0.92)$ \\
\hline
\end{tabular}

Note. SD: standard deviation.

stress, and anxiety (data averaged over all days can be found in Table 3).

We found statistically significant differences between days with and without masturbation and pornography use only for three patients (D, F, and G; Table 4). All of these had significantly lower mood on days with masturbation and pornography use. Additionally, patient D on average felt more tired, more stressed, and had a higher level of anxiety on days with masturbation and pornography use when compared to days without pornography and masturbation.

\section{Factors related to binges}

In contrast to regular pornography use, where most of the patients had difficulty in identifying triggering situations when asked (during the clinical interviews) for situations that triggered binge pornography use, the majority of patients reported stress, problems in personal life, and fear of failure in meeting high expectations of significant others as common factors. One person associated binges with work-related stress. Three subjects noticed that binges were related to the feeling of anger or loneliness and rejection.

All patients declared that during pornographic binges they initially experienced positive emotions (e.g., excitement and pleasure). Then, during the binge, most of the subjects do not have any specific thoughts ("cut off from thinking") and dissociate from their emotions. Just after the binge, they usually regret the wasted time or neglect of their duties. Such thoughts are accompanied by negative emotions, such as shame, feeling of loneliness, disgust, guilt, anger, sadness, anxiety, a sense of hopelessness, lack of self-respect, and depressed mood. Patients also feel irritation and anger. Five men reported having negative thoughts about themselves, e.g., "I am weak," "I could spend this time on so many hobbies, ideas, meetings with people instead of watching porn," and "I failed again." Three subjects did not report any specific thoughts after binges (Figure 3).

Diary assessment data were examined for differences between average levels of mood, tiredness, stress, and anxiety during the days with binges versus days without binges. This comparison uncovered many more significant differences than the previous one, related just to pornography viewing and masturbation (Table 4). For all but one subject $(\mathrm{G})$, pornographic binges led to either a decrease of mood (patients D, E, F, and H) or stress (patients A, D, and E). Just after the binge, they usually have thoughts about wasted time or neglected duties. Such thoughts are accompanied by negative emotions, such as shame, feelings of loneliness, disgust, guilt, anger, sadness, anxiety, a sense of hopelessness, lack of self-respect, and depressed mood."

Finally, we examined the potential for a causal relation between variables assessed with diaries (mood, tiredness, stress, and anxiety) and binge PuM (Table 5). For this purpose, similar to the previous analysis (presented in Table 4), we selected days with binge PuM (as defined in "Methods" section) and days without binges. Then, we calculated differences in mood, tiredness, stress, and anxiety between days immediately preceding a "binge day" and days "without a binge" (Supplementary Table S4) and days immediately following a "binge day" and days "without a binge" (Supplementary Table S5). Figure 4 presents a number of significant differences for each of these two comparisons. A high number of significant differences for the days preceding binges would provide evidence for the hypothesis that decreased mood, high tiredness, stress, and anxiety may play a causal role in binge PuMs, while a high number of differences in days following binges would suggest that decreased mood, increased tiredness, stress, and anxiety may be the consequences of binge PuM.

There was no significant difference $\left[\chi^{2}=2.64, p=.104\right.$; calculated for proportions of significant/non-significant differences for days preceding binges (Supplementary Table S4) and following binges (Supplementary Table S5)] between the number of significant results from the analysis of the days after a binge and such analysis of the days followed by a binge (Figure 4 ).

\section{DISCUSSION AND CONCLUSIONS}

In this study, we interviewed nine patients seeking treatment for problematic PuM. We then collected questionnaire data and used a 10-week-long diary assessment to examine how the subjects describe factors related to their problematic sexual activity and how it corresponds to data collected in the diary assessment.

Both self-reported and diary data show that despite the fact of previous treatments, all individuals met CSB criteria (Kafka, 2010), and that the most common problematic sexual behavior was PuM (similarly to the study by Reid, Li, Gilliland, Stein, \& Fong, 2011). The majority of them find it difficult to identify specific triggers of pornography use; however, they are able to identify repetitive patterns of pornography 
use - such as specific locations (e.g., home and work), times, and situations (e.g., being alone). Based on diary assessment data (mood, tiredness, stress, and anxiety), the majority of subjects found it very hard to find any correlates of such sexual activity. Perhaps specific episodes of PuM play either the role of behavior directed toward reducing natural sexual arousal or the role of a mechanism for coping with negative mood, stress, and anxiety. The occurrence of both over the period of 70 days of assessment may be a possible cause of the non-significant relation to diary assessment variables.

Interestingly, seven out of nine subjects reported that, in the course of their life, they experienced binge PuM lasting multiple hours and happening multiple times a day. In the case of such binges, the majority of subjects were able to indicate a number of triggers. Among the most commonly mentioned were stress, problems in personal life, fear of failure in meeting high expectations of significant others, anger, and feeling of loneliness and rejection. Similar findings were reported previously by Reid, Li, et al. (2011) who showed that pornography use was related to several negative outcomes, such as loneliness and anxiety. These complex cognitive and emotional states can be related to the simpler variables measured in diaries. We checked this hypothesis, and indeed, diary assessment data showed a significant relation between binges and decreased mood and increased stress and anxiety for all individuals in our group, except one.

According to the patients, binge pornography use allows them to feel excitement and pleasure and helps to "turn off thinking and emotions." Such outcomes may be experienced as an effective short-term coping mechanism. Unfortunately, immediately after the binge, all subjects experienced negative emotions (such as shame, feeling of loneliness, disgust, guilt, anger, sadness, anxiety, and a sense of hopelessness) and negative thoughts about themselves (e.g., "I am weak," "I waste my time," and "I failed again"); and according to patients, the experience of binge is related to the feeling of having lost control over one's own behavior.

Previous studies have shown that this feeling of the loss of control may be a crucial factor leading to treatment-seeking behavior among men (Gola, Lewczuk, et al., 2016) and women (Lewczuk et al., 2017). Despite the fact that binge PuM seems to be very common among CSB patients, very little is known about the characteristics and functions of these binges, as well as their mechanisms. The existence of binge pornography use was mentioned in many clinical observations and reported in a study by Reid, Stein, et al. (2011), but according to our knowledge, this is the first report attempting to focus on binges and examine the nature of these phenomena. Although we are aware of the preliminary character of our data (discussed further in the "Limitations" section) and of the need for more extensive research, we have been able to study several interesting aspects of binge PuM.

First, binge PuM may have different forms. According to the collected self-report data, binges may have the form of either continuous pornography watching accompanied by multiple masturbations for a few hours in a row (usually $>6 \mathrm{hr}$ with breaks lasting less than $30 \mathrm{~min}$ ) or multiple episodes (more than four times a day, lasting $0.5-1 \mathrm{hr}$ each) 


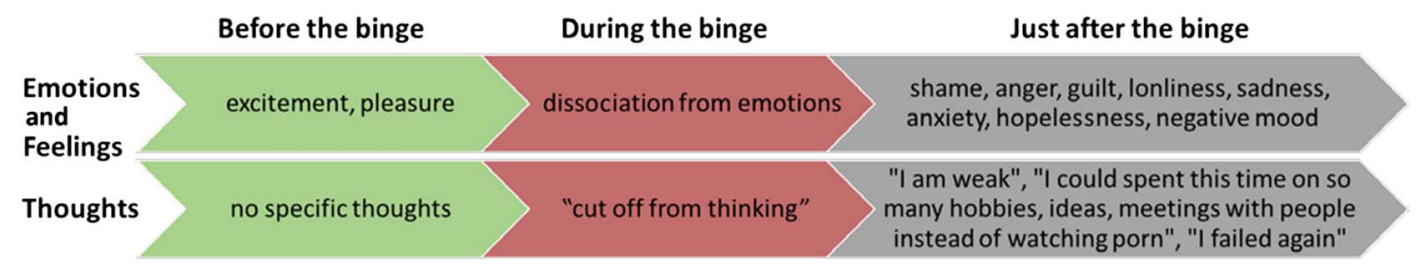

Figure 3. Self-reported emotions and thoughts before, during, and just after a pornographic binge

of pornography viewing in a single day, accompanied by masturbation.

Second, binge PuM seems to appear as a response to distressing situations and do not have the function of mere reduction of sexual arousal, but rather reduction of temporal tension, stress, or anxiety. It is unclear why a singular event of PuM is insufficient to ensure such emotional relief but escalates into a binge instead. We have a few non-exclusive and somewhat speculative hypotheses, worth investigating in future studies.

One possible explanation is that negative thoughts (e.g., "I failed again") and feelings (e.g., anger) after the first episode of PuM generate distress, which needs to be reduced through subsequent repetition of the same action, similarly to the mechanism of compulsive distress-reducing behavior appearing as a result of obsessive thoughts in OCD (Stein, 2002).

The second explanation is related to the recent finding (Gola, Wordecha, et al., 2017) that individuals seeking treatment for problematic pornography use have higher reactivity of the reward system in the brain (specifically the ventral striatum) in response to cues associated with pornography use. Perhaps one episode of PuM may temporarily sensitize this mechanism, increasing reactivity to subsequent cues and resulting with stronger urges, leading to binges.

The third explanation considers one of the mechanisms of addictive disorders related to habituation. Substance addiction models postulate decreased experience of pleasure during addiction development as an effect of habituation for rewards (Volkow et al., 2010). Such habituation leads to increased doses. In the case of CSB, the ultimate reward is the climax (Gola, Wordecha, Marchewka, et al., 2016); and in most solitary sexual behaviours, pornography provides the stimulation necessary for masturbation to end with climax (as shown in Figure 2, the vast majority of episodes of masturbation were accompanied by pornography use). It is possible that for CSB individuals, most erotic content is insufficient for climax and it takes more time to find novel, sufficiently arousing stimuli. It is also possible that after one climax there is a higher threshold for subsequent experiences, and longer pornography viewing is needed to find sufficiently arousing stimulation.

The fourth potential scenario assumes that the climax itself may not be the most pleasurable aspect of solitary sexual activity for some individuals with CSB. As it has been postulated (Gola, Wordecha, Marchewka, et al., 2016), visual sexual stimuli can themselves be a source of pleasure. To watch them, people are willing to invest effort comparable to that needed to obtain monetary gains (Sescousse, Caldú, Segura, \& Dreher, 2013). Interestingly, visual sexual stimuli evoke sexual arousal which is related to a further increase in motivation to watch them and to engage in sexual activity, up to the point of climax. After that, both sexual arousal and motivation to watch sexual stimuli decrease. We hypothesize that if CSB subjects experience climax as less pleasurable than the average person (i.e., due to habituation), they may focus more on pornography watching - which is a source of pleasure - and try to delay climax, which leads to long sessions of pornography use. We believe that all four mechanisms may contribute together to binge PuM, and each of them is worth more detailed studies.

Finally, we asked if decreased mood or increased tiredness, stress, and anxiety measured in diary assessment cause or are a consequence of binge pornography use. As we did not obtain clear results, this question needs further investigation. However, based on our data, we offer some suggestions. We observed that both decreased mood and increased tiredness appear one day before and one day after the binge. Therefore, it is possible that decreased mood and increased tiredness are both a cause and a consequence. When increased anxiety and stress happen one day after the binge, and more likely are consequence (Figure 4). Importantly, subjects exhibited very large inter-individual differences in the factors preceding and following binges. Thus, we think that binges may play a slightly different role for each of the individuals, helping one to cope with mood, another with fatigue, and result in different consequences, depending on individual cognitive beliefs. This variability indicates interesting potential significance of binges for clinical practice.

\section{Clinical significance}

Based on our results, we propose to discuss episodes of binge PuM in clinical work with CSB patients. According to the data presented in this study, the majority of CSB patients experience such binges. Interestingly, in contrast to individuals who experience regular episodes of short pornography use and single masturbation sessions, and have trouble identifying thoughts, feelings, and situations that lead to pornography use, individuals who experience binges are able to identify their automatic thoughts and emotions related to binges. This could be a good anchor for a cognitive and behavioral treatment. Also, the longitudinal diary assessment data show many more correlates of binges with mood, tiredness, stress, and anxiety changes, which support our observations derived from the data collected during interviews.

Another clinically significant aspect of binges is related to the potentially high variability of binge functions. It appears that such binges always play the role of coping mechanism, rather than an activity which reduces sexual 


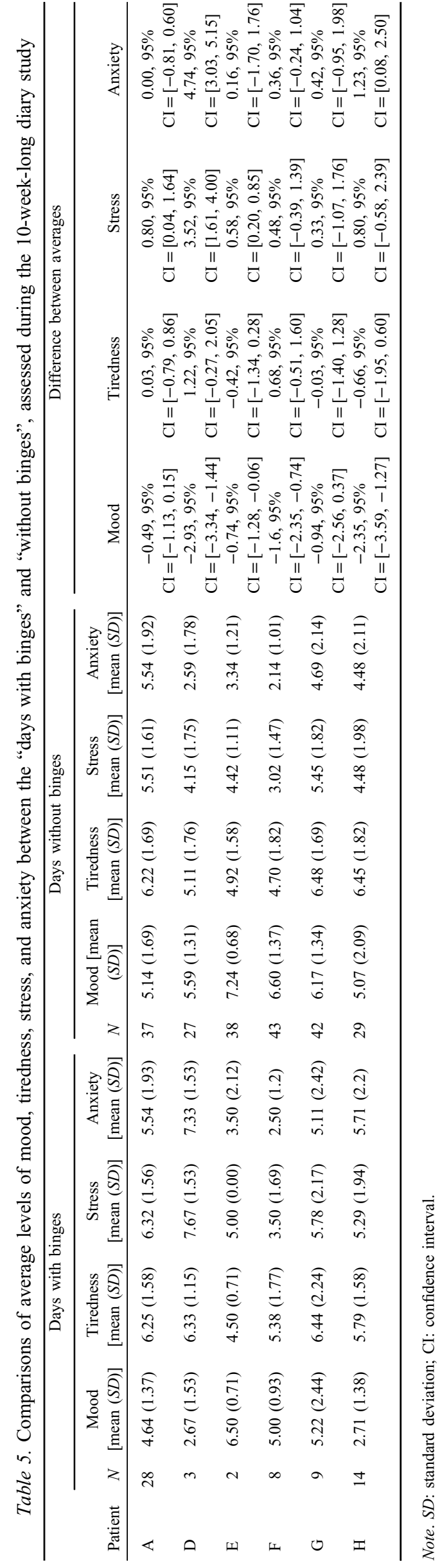

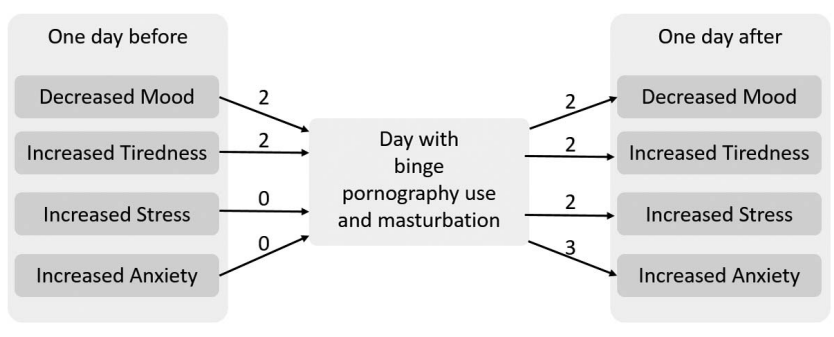

Figure 4. The number of subjects for whom we observed significant differences in mood, tiredness, stress, and anxiety (assessed with diaries) between days preceding a day with binge or a day without pornography and masturbation (left side of the figure; for exact differences see Supplementary Table S4). On the right side, we present the number of subjects for whom differences between days following a day with binge versus a day without PuM were significant (for exact differences, see Supplementary Table S5)

tension. Thus, a detailed analysis of binges (rather than analysis of any episodes of pornography use) may provide a faster path for identifying the life areas that need development of other, more adaptive coping mechanisms, and which may also need more attention in treatment.

Finally, one could ask if binge PuM should be included in diagnostic criteria of CSB proposed for the upcoming ICD-11 (WHO, 2018). However, while our small sample study shows that the majority of individuals who meet Kafka's (2010) CSB criteria experience binges, not all of them do so. Two out of nine subjects (B and $C$ ) never experienced binge $\mathrm{PuM}$, and one $(\mathrm{C})$ experienced it only a few times in his life. For this reason, we are opposed to inclusion of binge PuM as a criterion of CSB, but we also think that a detailed analysis of this symptom may be a source of valuable information for clinicians.

Another interesting and clinically relevant observation is related to patient B, who abstained from PuM for over 6 months (he reported daily sexual activity with his spouse), and yet was actively seeking treatment for CSB, citing pornography use as the main reason. He also met all the criteria proposed for ICD-11, which shows that despite temporal absence of most problematic behaviors, some people may still qualify for CSB disorder diagnosis, as there is no criterion defining maximal time elapsed since the last episode of CSB. We decided to include Subject B in this research report to show the full sample and to show that some individuals seek treatment and meet diagnostic criteria despite current absence of symptoms.

\section{Limitations}

We view this study as a preliminary investigation that may inspire other researchers to probe the nature, mechanisms, and role of binge PuM. It has a number of limitations and attempts to replicate it should definitely be made (we will be happy to share all our methodology with anyone interested in using it). First, we studied only nine individuals and only seven provided full data. Second, these individuals were actively seeking treatment for CSB and eight of them had tried CSB treatment before, so they were highly motivated to limit their pornography use. Third, all of them started treatment during our 70-day diary assessments and 
completed at least six sessions (usually on a weekly basis). This may significantly influence the collected diary data, and we suspect that it resulted in a much smaller number of CSBs than we could observe in CSB populations that were never treated. It can also result in higher self-awareness than that in individuals who received no treatment.

Further limitations are related to data quality and analysis. We did our best to collect high quality data during the diary assessments, but there were inevitable gaps in the data (Supplementary Table S2). We suspect that many episodes of sexual activity may have happened on the days when no diary entry was made, and that relapses may have been related to decreased motivation to persevere with the diary. There was no way for us to control this issue in this study. If this is indeed true, then the data on sexual activity are underreported. We asked patients to make one entry in the diary every day. Such a temporal resolution seems to be insufficient to determine a causal relation between variables, such as mood, anxiety, stress, etc., on one hand, and binges on the other. For future studies, we suggest ecological momentary assessments a few times a day as a better way to determine the causal relation and to avoid gaps in the data.

Due to the aforementioned limitations (potentially lower than usual sexual activity related to treatment and missing data), for the purposes of data analysis, we defined binge episodes as more than $1 \mathrm{hr}$ of pornography use and/ or 2 or more masturbations a day. We know from other studies that such a definition may overlap with the sexual activity of individuals who do not meet CSB criteria (Brand et al., 2016). Thus, for future studies on populations without treatment and with more advanced methodology (i.e., ecological momentary assessment), as well as for clinical purposes, we suggest that a binge be defined as a $2+$ hr of pornography use and/or 3+ masturbation sessions a day. We also encourage researchers to determine these thresholds in empirical studies.

Funding sources: This study was supported by the Polish National Science Centre, by two grants OPUS grant (2014/15/B/HS6/03792) (MG, MWi, MWo, and EK) and PRELUDIUM grant (2016/23/N/HS6/02906) (MWo). MG is additionally supported by scholarships of the Polish Ministry of Science scholarships (469/STYP/10/2015) and the Kosciuszko Foundation.

Authors' contribution: MWo contributed to study and methods design, subjects recruitment, conducting interviews, data analysis and interpretation, and manuscript writing. MWi contributed to data analysis and interpretation and manuscript preparation. EK contributed to questionnaires development. MS and AŁ contributed to development of diaries assessment software and data preprocessing. MG also contributed to study and methods design, data interpretation, manuscript writing, obtaining funding, and study supervision.

Conflict of interest: The authors report no conflict of interest with respect to the content of this manuscript.

\section{REFERENCES}

Brand, M., Snagowski, J., Laier, C., \& Maderwald, S. (2016). Ventral striatum activity when watching preferred pornographic pictures is correlated with symptoms of Internet pornography addiction. Neuroimage, 129, 224-232. doi:10.1016/ j.neuroimage.2016.01.033

Carnes, P., Green, B., \& Carnes, S. (2010). The same yet different: Refocusing the Sexual Addiction Screening Test (SAST) to reflect orientation and gender. Sexual Addiction \& Compulsivity, 17(1), 7-30. doi:10.1080/10720161003604087

Carpenter, B. N., Reid, R. C., Garos, S., \& Najavits, L. M. (2013). Personality disorder comorbidity in treatment-seeking men with hypersexual disorder. Sexual Addiction \& Compulsivity, 20, 79-90. doi:10.1080/10720162.2013.772873

Foa, E., Huppert, J., Leiberg, S., Langner, R., Kichic, R., Hajcak, G., \& Salkovskis, P. M. (2002). The Obsessive-Compulsive Inventory: Development and validation of a short version. Psychological Assessment, 14(4), 485-496. Retrieved from http://psycnet.apa.org/journals/pas/14/4/485/

Gola, M., Kowalewska, E., Wierzba, M., Wordecha, M., \& Marchewka, A. (2015). Polska adaptacja Kwestionariusza Pobudliwości Seksualnej SAI-PL i walidacja w grupie mężczyzn [Polish adaptation of the Sexual Arousability Inventory SAI-PL and validation for the males]. Psychiatria, 12(4), 245-254.

Gola, M., Lewczuk, K., \& Skorko, M. (2016). What matters: Quantity or quality of pornography use? Psychological and behavioral factors of seeking treatment for problematic pornography use. The Journal of Sexual Medicine, 13(5), 815-824. doi:10.1016/j.jsxm.2016.02.169

Gola, M., Miyakoshi, M., \& Sescousse, G. (2015). Sex, impulsivity, and anxiety: Interplay between ventral striatum and amygdala reactivity in sexual behaviors. Journal of Neuroscience, 35(46), 15227-15229. doi:10.1523/JNEUROSCI. 3273-15.2015

Gola, M., \& Potenza, M. N. (2016). Paroxetine treatment of problematic pornography use: A case series. Journal of Behavioral Addictions, 5(3), 529-532. doi:10.1556/2006.5.2016.046

Gola, M., \& Potenza, M. N. (2018). The proof of the pudding is in the tasting: Data are needed to test models and hypotheses related to compulsive sexual behaviors. Archives of Sexual Behavior. Advance online publication. 1-3. doi:10.1007/ s10508-018-1167-x.

Gola, M., \& Potenza, M. N. (in press). Compulsive sexual behavior disorder in the ICD-11- promoting educational, classification, treatment and policy initiatives. Journal of Behavioral Addictions.

Gola, M., Skorko, M., Kowalewska, E., Kołodziej, A., Sikora, M., Wodyk, M., Wodyk, Z., \& Dobrowolski, P. (2017). Sexual Addiction Screening Test-polska adaptacja. [Polish adaptation of Sexual Addiction Screening Test]. Psychiatria Polska, 51(1), 95-115. doi:10.12740/PP/OnlineFirst/61414

Gola, M., Wordecha, M., Marchewka, A., \& Sescousse, G. (2016). Visual sexual stimuli-cue or reward? A perspective for interpreting brain imaging findings on human sexual behaviors. Frontiers in Human Neuroscience, 10, 402. doi:10.3389/ fnhum.2016.00402

Gola, M., Wordecha, M., Sescousse, G., Lew-Starowicz, M., Kossowski, B., Wypych, M., Makeig, S., Potenza, M. N., \& 
Marchewka, A. (2017). Can pornography be addictive? An fMRI study of men seeking treatment for problematic pornography use. Neuropsychopharmacology, 42(10), 2021-2031. doi:10.1038/npp.2017.78.

Kafka, M. P. (2010). Hypersexual disorder: A proposed diagnosis for DSM-V. Archives of Sexual Behavior, 39(2), 377-400. doi:10.1007/s10508-009-9574-7

Kashdan, T. B., Adams, L. M., Farmer, A. S., Ferssizidis, P., McKnight, P. E., \& Nezlek, J. B. (2013). Sexual healing: Daily diary investigation of the benefits of intimate and pleasurable sexual activity in socially anxious adults. Archives of Sexual Behavior, 43(7), 1417-1429. doi:10.1007/s10508013-0171-4

Kirby, K. N., \& Maraković, N. N. (1996). Delay-discounting probabilistic rewards: Rates decrease as amounts increase. Psychonomic Bulletin \& Review, 3(1), 100-104. doi:10.3758/ BF03210748

Klucken, T., Wehrum-Osinsky, S., Schweckendiek, J., Kruse, O., \& Stark, R. (2016). Altered appetitive conditioning and neural connectivity in subjects with compulsive sexual behavior. The Journal of Sexual Medicine, 13(4), 627-636. doi:10.1016/j. jsxm.2016.01.013

Kor, A., Fogel, Y. A., Reid, R. C., \& Potenza, M. N. (2013). Should hypersexual disorder be classified as an addiction?. Sexual Addiction \& Compulsivity, 20(1-2), 27-47. doi:10. 1080/10720162.2013.768132

Kraus, S. W., Gola, M., Kowalewska, E., Lew-Starowicz, M., Hoff, R. A., Porter, E., \& Potenza, M. N. (2017). Brief pornography screener: A comparison of US and Polish pornography users. Journal of Behavioral Addictions, 6(S1), 27-28.

Kraus, S. W., Krueger, R. B., Briken, P., First, M. B., Stein, D. J., Kaplan, M. S., Voon, V., Abdo, C. H., Grant, J. E., Atalla, E., \& Reed, G. M. (2018). Compulsive sexual behaviour disorder in the ICD-11. World Psychiatry, 17(1), 109-110. doi:10.1002/ wps.20499

Kraus, S. W., Voon, V., \& Potenza, M. N. (2016a). Neurobiology of compulsive sexual behavior: Emerging science. Neuropsychopharmacology, 41(1), 385-386. doi:10.1038/npp. 2015.300

Kraus, S. W., Voon, V., \& Potenza, M. N. (2016b). Should compulsive sexual behavior be considered an addiction? Addiction, 111(12), 2097-2106. doi:10.1111/add.13297

Lewczuk, K., Szmyd, J., Skorko, M., \& Gola, M. (2017). Treatment seeking for problematic pornography use among women. Journal of Behavioral Addictions, 6(4), 445-456. doi:10.1556/ 2006.6.2017.063

Ley, D., Prause, N., \& Finn, P. (2014). The emperor has no clothes: A review of the 'pornography addiction' model. Current Sexual Health Reports, 6(2), 94-105. doi:10.1007/s11930-014-0016-8

Potenza, M. N., Gola, M., Voon, V., Kor, A., \& Kraus, S. W. (2017). Is excessive sexual behaviour an addictive disorder? The Lancet Psychiatry, 4(9), 663-664. doi:10.1016/S22150366(17)30316-4

Prause, N., Janssen, E., Georgiadis, J., Finn, P., \& Pfaus, J. (2017). Data do not support sex as addictive. The Lancet Psychiatry, 4(12), 899. doi:10.1016/S2215-0366(17)30441-8
Reid, R. C., Garos, S., \& Carpenter, B. N. (2011). Reliability, validity, and psychometric development of the Hypersexual Behavior Inventory in an outpatient sample of men. Sexual Addiction \& Compulsivity, 18(1), 30-51. doi:10.1080/ 10720162.2011.555709

Reid, R. C., Li, D. S., Gilliland, R., Stein, J. A., \& Fong, T. (2011). Reliability, validity, and psychometric development of the Pornography Consumption Inventory in a sample of hypersexual men. Journal of Sex \& Marital Therapy, 37(5), 359-385. doi:10.1080/0092623X.2011.607047

Reid, R. C., Stein, J. A., \& Carpenter, B. N. (2011). Understanding the roles of shame and neuroticism in a patient sample of hypersexual men. The Journal of Nervous and Mental Disease, 199(4), 263-267. doi:10.1097/NMD.0b013e3182 $125 \mathrm{~b} 96$

Reid, R. C., Temko, J., Moghaddam, J. F., \& Fong, T. W. (2014). Shame, rumination, and self-compassion in men assessed for hypersexual disorder. Journal of Psychiatric Practice, 20(4), 260-268. doi:10.1097/01.pra.0000452562.98286.c5

Rolland, B., \& Naassila, M. (2017). Binge drinking: Current diagnostic and therapeutic issues. CNS Drugs, 31(3), 181-186. doi:10.1007/s40263-017-0413-4

Sescousse, G., Caldú, X., Segura, B., \& Dreher, J.-C. (2013). Processing of primary and secondary rewards: A quantitative meta-analysis and review of human functional neuroimaging studies. Neuroscience and Biobehavioral Reviews, 37(4), 681-696. doi:10.1016/j.neubiorev.2013.02.002

Stein, D. J. (2002). Obsessive-compulsive disorder. The Lancet, 360(9330), 397-405. doi:10.1016/S0140-6736(02)09620-4

Stein, D. J., Black, D. W., Shapira, N. A., \& Spitzer, R. L. (2001). Hypersexual disorder and preoccupation with Internet pornography. American Journal of Psychiatry, 158(10), 1590-1594. doi:10.1176/appi.ajp.158.10.1590

Sosnowski, T., \& Wrześniewski, K. (1983). Polska adaptacja inwentarza STAI do badania stanu i cechy lęku [Polish adaptation of STAI Inventory for state and trait anxiety assessment]. Przegl d Psychologiczny, 26, 393-412.

Volkow, N. D., Wang, G.-J., Fowler, J. S., Tomasi, D., Telang, F., \& Baler, R. (2010). Addiction: Decreased reward sensitivity and increased expectation sensitivity conspire to overwhelm the brain's control circuit. BioEssays, 32(9), 748-755. doi:10.1002/bies.201000042

Voon, V., Mole, T. B., Banca, P., Porter, L., Morris, L., Mitchell, S., Lapa, T. R., Karr, J., Harrison, N. A., Potenza, M. N., \& Irvine, M. (2014). Neural correlates of sexual cue reactivity in individuals with and without compulsive sexual behaviours. PLoS One, 9(7), e102419. doi:10.1371/journal. pone.0102419

World Health Organization [WHO]. (2018). The ICD-11 classification of mental and behavioural disorders: Clinical descriptions and diagnostic guidelines. Retrieved from https://icd.who.int/dev11/1-m/en\#/http://id.who.int/icd/entity/ 1630268048

Zigmond, A. S., \& Snaith, R. P. (1983). The Hospital Anxiety and Depression Scale. Acta Psychiatrica Scandinavica, 67(6), 361-370. doi:10.1111/j.1600-0447.1983.tb09716.x 